\title{
Influence of Expression Plasmid of Connective Tissue Growth Factor and Tissue Inhibitor of Metalloproteinase-1 shRNA on Hepatic Precancerous Fibrosis in Rats
}

\author{
Qun Zhang', Fu-li Shu², Yu-Feng Jiang'*, Xin-En Huang ${ }^{3 *}$
}

\begin{abstract}
Background: In this study, influence caused by expression plasmids of connective tissue growth factor (CTGF) and tissue inhibitor of metalloproteinase-1 (TIMP-1) short hairpin RNA (shRNA) on mRNA expression of CTGF,TIMP-1,procol- $\alpha 1$ and PCIII in hepatic tissue with hepatic fibrosis, a precancerous condition, in rats is analyzed. Materials and Methods: To screen and construct shRNA expression plasimid which effectively interferes RNA targets of CTGF and TIMP-1 in rats. 50 cleaning Wistar male rats are allocated randomly at 5 different groups after precancerous fibrosis models and then injection of shRNA expression plasimids. Plasmid psiRNA-GFP-Com (CTGF and TIMP-1 included), psiRNA-GFP-CTGF, psiRNA-GFP-TIMP-1 and psiRNADUO-GFPzeo of blank plasmid are injected at group A, B, C and D, respectively, and as model control group that none plasimid is injected at group E. In 2 weeks after last injection, to hepatic tissue at different groups, protein expression of CTGF, TIMP-1, procol- $\alpha 1$ and PC III is tested by immunohistochemical method and,mRNA expression of CTGF,TIMP-1,procol-a1 and PCIII is measured by real-time PCR. One-way ANOVA is used to comparison between-groups. Results: Compared with model group, there is no obvious difference of mRNA expression among CTGF,TIMP-1,procol- $\alpha$ 1,PC III and of protein expression among CTGF, TIMP-1, procol- $\alpha 1$, PC III in hepatic tissue at group injected with blank plasmid. Expression quantity of mRNA of CTGF, TIMP-1, procol- $\alpha 1$ and PCIII at group A, B and C decreases, protein expression of CTGF, TIMP-1, procol- $\alpha 1$, PC III in hepatic tissue is lower, where the inhibition of combination RNA interference group (group A) on procol- $\alpha 1$ mRNA transcription and procol- $\alpha 1$ protein expression is superior to that of single interference group (group B and $C)(P<0.01$ or $P<0.05)$. Conclusions: RNA interference on CTGF and/or TIMP-1 is obviously a inhibiting factor for mRNA and protein expression of CTGF, TIMP-1, procol- $\alpha 1$ and PCIII. Combination RNA interference on genes of CTGF and TIMP-1 is superior to that of single RNA interference, and this could be a contribution for prevention of precancerous condition.
\end{abstract}

Keywords: RNA interference - connective tissue growth factor - tissue inhibitor of metalloproteinase-1

Asian Pac J Cancer Prev, 16 (16), 7205-7210

\section{Introduction}

Many past studies suggested that transforming growth factor $\beta$ (TGF- $\beta 1$ ) is the strongest factor prompting hepatic fibrosis in the activation of hepatic stellate cell (HSC) that other cell-factors also participated in, block of signal pathway of TGF- $\beta 1$ is considered to be the best option for hepatic fibrosis therapy (Lee et al., 2011). RNA interference technology is used to block CTGF and TIMP-1 of downstream molecule. It may provide a good foundation to explore new therapies of hepatic fibrosis based on two ways including block of CTGF during forming extracellular matrix (ECM) and removal of inhibition by TIMP-1 on EMC degradation (to promote degradation).
This study uses recombinant plasmids constructed previously with CTGFshRNA and TIMP-1shRNA respectively of psiRNA-GFP-CTGF, psiRNA- GFPTIMP-1 and psiRNA- GFP-Com (CTGF and TIMP1included), to transfect rats with hepatic fibrosis. And further analyzes their influence on mRNA and proteinrelated expression of CTGF and TIMP-1, to prepare to study the roles of CTGF and TIMP- 1 of TGF- $\beta 1$ downstream effect medium in hepatic fibrosis and explore the gene therapy for hepatic fibrosis.

\section{Materials and Methods}

Materials

The recombinant plasmids, namely, psiRNA- GFP- 
Com (CTGF and TIMP-1 in), psiRNA-GFP- CTGF and psiRNA-GFP-TIMP-1 were constructed in our laboratory (Jiang et al., 2011; Jiang et al., 2011; Jiang et al., 2012). Target sequences: CTGF1560-1580nt and TIMP-1412432. Other reagents were purchased from chemical companies and applied in line with the instructions. Cleaning ${ }^{\top}$ Wistar rats weighed from $140 \mathrm{~g}-160 \mathrm{~g}$ were purchased from ChengDu DaShuo Animal Technology Co., Ltd (License No.SCXK (Chuan) 2008-24) .

\section{Methods}

Modeling and groups of hepatic fibrosis in rats: 50 Wistar male rats were accepted intraperitoneal injection of solution of $1 \% \mathrm{DMN}$ with $80 \mu \mathrm{l} / 100 \mathrm{~g}$ for 3 weeks, 3 times per week. Rats were weighed 1 time per week for adjustment of drug dosage according to the weight. 15 rats were gone after 3 weeks. Hepatic fibrosis was seen in condition of microscope after HE stained for dead rats' hepatic tissues. The living rats were allocated randomly at group A, B, C, D and E, 7 rats per group, respectively. Group A: dual RNA interference (injected with psiRNAGFP -Com of transfection plasmid), group B: pshRNACTGF interference (injected with psiRNA-GFP-CTGF), group C: pshRNA-TIMP-1 interference (injected with psiRNA-GFP-TIMP-1), group D: control group of blank plasmid (injected with psiRNA-DUOGFPzeo), group E: model control group (injected with nothing).

The plasmids were obtained according to the instructional manual of EndoFree Plasmid Giga Kit of QIAGEN Company. At different groups, the rats were injected with plasmid. The plasmid dosage of $100 \mu \mathrm{g} / 100 \mathrm{~g}$ was dissolved in Ringer's solution of $6 \%$ rat's weight, rapid caudal vein injection in $15 \mathrm{~s}$ was done to the rats for totally 4 times, 1 time 2 weeks. Each group lost 2 rats one after another (totally 10 rats) during the injection. The rats were killed and livers were obtained in 2 weeks after last injection.

$\underline{\text { RNA interference plasmid injected in hepatic fibrosis }}$ in rats at different groups: The plasmids were obtained according to the instructional manual of EndoFree Plasmid Giga Kit of QIAGEN Company. At different groups, the rats were injected with plasmid. The plasmid dosage of $100 \mu \mathrm{g} / 100 \mathrm{~g}$ was dissolved in Ringer's solution of $6 \%$ rat's weight, rapid caudal vein injection in $15 \mathrm{~s}$ was done to the rats for totally 4 times, 1 time 2 weeks. Each group lost 2 rats one after another (totally 10 rats) during the injection. The rats were killed and livers were obtained in 2 weeks after last injection.

Classifications of fibrosis of hepatic histology:
Hepatic frozen section was carried out to observe green fluorescence protein (GFP) expression in condition of fluorescence microscope. Paraffin section and HE stained after successful transfection were completed, and film reading was done by two professional pathologists after unified standard. Stages and semi-quantitative scoring system (SSS) of hepatic fibrosis were done by reference to the consensus about evaluating hepatic fibrosis diagnosis and efficacy issued by Hepatic Fibrosis Group, a part of Chinese Society of Hepatology (Hepatic et al., 2002). Stages of hepatic fibrosis degrees: S0: no fibrosis; S1: enlargement of fibrosis in portal area, peri-sinusoid and partial lobular fibrosis; S2: fibrosis around portal area, formation of fibrous septum, maintenance of lobular structures. S3: disordered lobular structures of fibrous septum, no cirrhosis; S4: earlier cirrhosis; SSS of fibrosis was given by Table 2 .

Hepatic Protein Expression of CTGF, TIMP-1, procol- $\alpha 1$ and PC III at Different Groups were Tested Using Immumohistochemica Method.

Hepatic mRNA Expression of CTGF, TIMP-1. Procol- $\alpha 1$ and PC III at Different Groups were Tested Using Real-Time PCR: The hepatic total RNA was obtained and was reversely transcribed to be cRNA, target DNA was amplified by FQ-PCR with Eva Green relative quantitative measurement. Standard curves of tested genes were established respectively. Analysis of Bio-Rad CFX96 software according to standard curves and $\mathrm{Ct}$ value of PCR was carried out, based on Pfaffl method, gene expression=

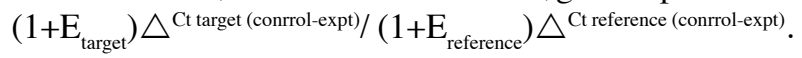

\section{Statistical analysis}

Measurement data were expressed as the means \pm standard error and the data were analyzed by SPSS software version 17.0. Data with normal distribution and homoscedasticity were analyzed by one-way ANOVA, and with heteroscedasticity were tested by rank sum test. A p-value $<0.05$ was considered to be significant.

Table 2. Comparison of Stage and Scoring of Liver Fibrosis at Different Groups $(\overline{\mathbf{x}} \pm \mathbf{s})$

\begin{tabular}{lrrrrrrrc}
\hline Group & $\mathrm{n}$ & \multicolumn{4}{c}{ Stage } & & \multicolumn{2}{c}{ Scoring } \\
& & 0 & 1 & 2 & 3 & 4 & $(\overline{\mathrm{x}} \pm \mathrm{s})$ \\
\hline A Dual Interference & 5 & 0 & 3 & 1 & 1 & 0 & $3.5 \pm 1.12$ \\
B CTGF Interference & 5 & 0 & 2 & 2 & 1 & 0 & $4.1 \pm 1.52$ \\
C TIMP-1 Interference & 5 & 0 & 1 & 4 & 0 & 0 & $4.1 \pm 0.55$ \\
D Blank Plasimid Interference & 5 & 0 & 0 & 0 & 2 & 3 & $8.4 \pm 2.51$ \\
E Model Control Group & 5 & 0 & 0 & 0 & 3 & 2 & $7.6 \pm 1.34$ \\
\hline
\end{tabular}

Compared with group $\mathrm{E}, \mathrm{P}<0.01$

Table 1. The Fibrosis semi-quantitative scoring system (SSS)

\begin{tabular}{|c|c|c|c|c|}
\hline \multirow[b]{2}{*}{ Scoring } & Lobule (L) & \multirow[t]{2}{*}{ Portal area $(\mathrm{P})$} & \multicolumn{2}{|c|}{ Fibrous septum*, } \\
\hline & Around vein/Peri-sinusoid & & Number(N) & Width(W) \\
\hline 0 & Normal & Normal & 0 & -- \\
\hline 1 & partial fibrosis, little & enlargement of septa without fibrosis & 6 fibrous septa/10mm & fine/imcomplete \\
\hline 2 & diffuse fibrosis, much & enlargement of septa with fibrosis & 6 fibrous septa/10mm & loosen, wide \\
\hline 3 & -- & cirrhosis & cirrhosis & tight, wide \\
\hline 4 & -- & -- & & 2/3biopsy area \\
\hline
\end{tabular}

$* \mathrm{~L}+\mathrm{P}+2 \times(\mathrm{N} \times \mathrm{W}) ; *$ only one micro-fiber septum in sample, 0.5 of $\mathrm{W}$ scoring, septum width between the two, the scoring was expressed by means. 


\section{Results}

\section{Transfection of recombinant plasmid}

In 2 weeks after last plasmid injection for experimental rats, the hepatic tissues obtained from rats showed green fluorescent protein (GFP) at group A, B, C and D in condition of fluorescence microscope. Hepatic tissues at model control group showed nothing about GFP, which suggested successful transfection.

\section{Classifications of hepatic fibrosis}

After HE stained at group D and E, denatured and necrotic hepatic cells, enlargement of fibrosis, hyperplastic blood vessels and bile ducts in portal area, disordered lobular structures, fibrous septum between lobules and pseudo lobules in partial sections were seen in condition of microscope, stages of hepatic fibrosis included S3 and/or S4 (cvure 1 and Figure 2). At group A, B and C, however, enlargement of fibrosis, tiny peri-sinusoid and partial lobular fibrosis, slight disordered lobular structures in partial sections, formation of tiny lobular fibrous septum were seen in condition of microscope, but there was no formation of pseudo lobules, stages of hepatic fibrosis included S1 to S3 (Figure 3, Figure 4 and Figure 5), degree of fibrosis was slighter than group D and group E.

Histology of hepatic fibrosis changed at different groups to make semiquantitative scoring system (SSS) of

Table 2. Comparison of Stage and Scoring of Liver Fibrosis at Different Groups $(\overline{\mathbf{x}} \pm \mathbf{s})$

\begin{tabular}{lrrrrrrrc}
\hline Group & $\mathrm{n}$ & \multicolumn{3}{c}{ Stage } & & Scoring \\
& & 0 & 1 & 2 & 3 & 4 & $(\overline{\mathrm{x}} \pm \mathrm{s})$ \\
\hline A Dual Interference & 5 & 0 & 3 & 1 & 1 & 0 & $3.5 \pm 1.12$ \\
B CTGF Interference & 5 & 0 & 2 & 2 & 1 & 0 & $4.1 \pm 1.52$ \\
C TIMP-1 Interference & 5 & 0 & 1 & 4 & 0 & 0 & $4.1 \pm 0.55$ \\
D Blank Plasimid Interference & 5 & 0 & 0 & 0 & 2 & 3 & $8.4 \pm 2.51$ \\
E Model Control Group & 5 & 0 & 0 & 0 & 3 & 2 & $7.6 \pm 1.34$ \\
\hline
\end{tabular}

Compared with group $\mathrm{E}, \mathrm{P}<0.01$

Table 3. The Protein expression of PC I at the different groups $(\overline{\mathbf{x}} \pm \mathbf{s})$

\begin{tabular}{lcc}
\hline Group & Sample No.(n) & $\operatorname{Lg}(\mathrm{IOD})$ \\
\hline A Dual Interference & 5 & $2.98 \pm 0.76^{\&}$ \\
B CTGF Interference & 5 & $3.28 \pm 0.40^{*}$ \\
C TIMP-1 Interference & 5 & $3.30 \pm 0.57^{*}$ \\
D Blank Plasmid Interference & 5 & $3.75 \pm 0.66$ \\
E Model Control Group & 5 & $3.76 \pm 0.29$ \\
\hline
\end{tabular}

*Compared with group E, $\mathrm{P}<0.01$; \&Compared with group B, C, D and $\mathrm{E}, \mathrm{P}<0.05$

Table 4. The Protein Expression of PC III at the Different Groups ( $\overline{\mathbf{x}} \pm$ s)

\begin{tabular}{lcc}
\hline Group & Sample No.(n) & $\operatorname{Lg}(\mathrm{IOD})$ \\
\hline A Dual Interference & 5 & $3.58 \pm 0.27^{*}$ \\
B CTGF Interference & 5 & $3.65 \pm 0.35^{*}$ \\
C TIMP-1 Interference & 5 & $3.61 \pm 0.61^{*}$ \\
D Blank Plasmid Interference & 5 & $3.98 \pm 0.63$ \\
E Model Control Group & 5 & $4.02 \pm 0.83$ \\
\hline
\end{tabular}

*Compared with group E, $\mathrm{P}<0.01$ fibrosis, group E, D, A, B and C got scores of 7.6 \pm 1.34 , $8.4 \pm 2.51,3.5 \pm 1.12,4.1 \pm 1.52$ and $4.1 \pm 0.55$, respectively. There was no obvious difference between group D and $\mathrm{E}$, compared with group $\mathrm{E}$ that lower scores at group A, $\mathrm{B}, \mathrm{C}(P<0.01)$ and there was no obvious difference in multiple comparisons among group $\mathrm{A}, \mathrm{B}$ and $\mathrm{C}$, as given by Table 2 .

\section{Results of Hepatic Immumohistochemical Staining at Different groups}

Immumohistochemical Staining of Procol- $\alpha 1$ : Yellowbrown procol- $\alpha 1$ was observed in hepatic area around central vein, fibrous septum of portal area and infiltration area of inflammatory cells in rats at different groups. Integrated optical density (IOD)was measured by ImagePro Plus 6.0 (IPP6.0) and then semi-quantitative scoring system (SSS)analysis was made. Compared with group E, IOD of group D was no significant reduction and the difference was considered to be no significant. Compared with group D and E, IOD of group A, B and C was obviously lower $(P<0.01)$, however. Multiple comparisons among three recombinant plasmid interference groups, compared with group B and C, group A was lower $(P<0.01)$, there was no significant difference between group $\mathrm{B}$ and $\mathrm{C}(P>0.05)$, as given by Table 3 .

Immumohistochemical Staining of PC III: A mass of Yellow-brown streak PC III was observed in hepatic fibrous septum of portal area in rats at different groups. Compared with group E, difference of IOD of group D was considered to be no significant $(P>0.05)$. Compared with group D and E, IOD of group A, B and C was obviously lower $(P<0.01)$. Multiple comparisons among group A, B and $\mathrm{C}$, the difference was considered to be no significant $(P>0.05)$, as given by Table 4 .

Immumohistochemical Staining of CTGF: Yellowbrown CTGF protein was observed in hepatic area around central vein, fibrous septum of portal area and infiltration area of inflammatory cells in rats at different groups. Compared with group E, difference of IOD between group $\mathrm{D}, \mathrm{C}$ and group $\mathrm{E}$ was considered to be

Table 5. The Protein Expression of CTGF at the Different Groups $(\overline{\mathbf{x}} \pm \mathbf{s})$

\begin{tabular}{lcc}
\hline Group & Sample No.(n) & $\mathrm{Lg}(\mathrm{IOD})$ \\
\hline A Dual Interference & 5 & $2.93 \pm 0.74^{*}$ \\
B CTGF Interference & 5 & $3.79 \pm 0.66$ \\
C TIMP-1 Interference & 5 & $2.95 \pm 0.75^{*}$ \\
D Blank Plasmid Interference & 5 & $3.81 \pm 0.39$ \\
E Model Control Group & 5 & $3.76 \pm 0.47$ \\
\hline
\end{tabular}

*Compared with group E, $\mathrm{P}<0.01$

Table 6. The Protein Expression of TIMP-1 at the Different Groups $(\overline{\mathbf{x}} \pm \mathbf{s})$

\begin{tabular}{lcc}
\hline Group & Sample No.(n) & $\operatorname{Lg}(\mathrm{IOD})$ \\
\hline A Dual Interference & 5 & $3.21 \pm 0.27^{*}$ \\
B CTGF Interference & 5 & $3.87 \pm 0.42$ \\
C TIMP-1 Interference & 5 & $3.18 \pm 0.63^{*}$ \\
D Blank Plasmid Interference & 5 & $4.02 \pm 0.29$ \\
E Model Control Group & 5 & $3.94 \pm 0.40$ \\
\hline *Compared with group E, $\mathrm{P}<0.01$ &
\end{tabular}


Table 7. Gene Expression Quantity at Different Groups $(\overline{\mathbf{x}} \pm \mathbf{s})$

\begin{tabular}{lcccc}
\hline Group & CTGF & TIMP-1 & procol- $\alpha 1$ & PC III \\
\hline A Dual Interference & $1.13 \pm 0.34^{*}$ & $0.95 \pm 0.11^{*}$ & $1.05 \pm 0.25^{* *}$ & $1.44 \pm 0.42^{*}$ \\
B CTGF Interference & $1.21 \pm 0.37^{*}$ & $1.41 \pm 0.39$ & $1.53 \pm 0.77^{*}$ & $1.46 \pm 0.27^{*}$ \\
C TIMP-1 interference & $1.97 \pm 0.76$ & $0.84 \pm 0.12^{*}$ & $1.57 \pm 0.25^{*}$ & $1.27 \pm 0.28^{*}$ \\
D Blank Plasmid Interference & $1.85 \pm 0.41$ & $1.56 \pm 0.71$ & $2.15 \pm 0.46$ & $2.47 \pm 0.52$ \\
E Model Control Group & $1.93 \pm 0.34$ & $1.61 \pm 0.68$ & $2.17 \pm 0.45$ & $2.45 \pm 1.01$ \\
\hline
\end{tabular}

*Compared with group $\mathrm{E}, \mathrm{P}<0.01$ or $\mathrm{P}<0.01$ : **Compared with group $\mathrm{B}$ and $\mathrm{C}, \mathrm{P}<0.05$, Compared with group $\mathrm{D}$ and $\mathrm{E}, \mathrm{P}<0.01$

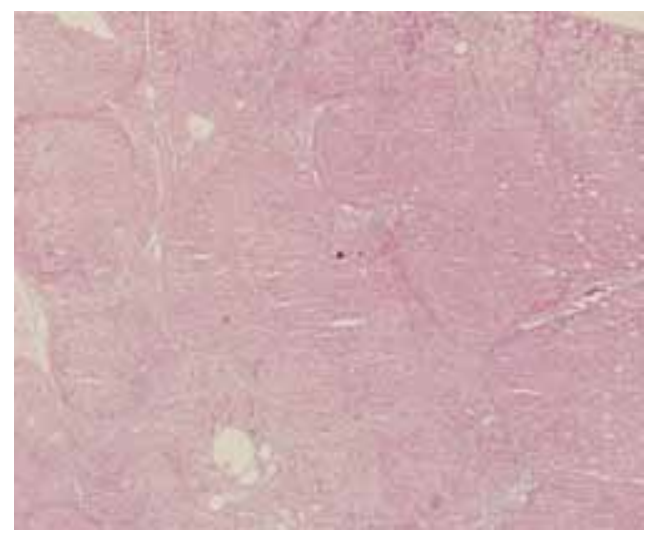

Figure 1. Group D (HE stained ×40)

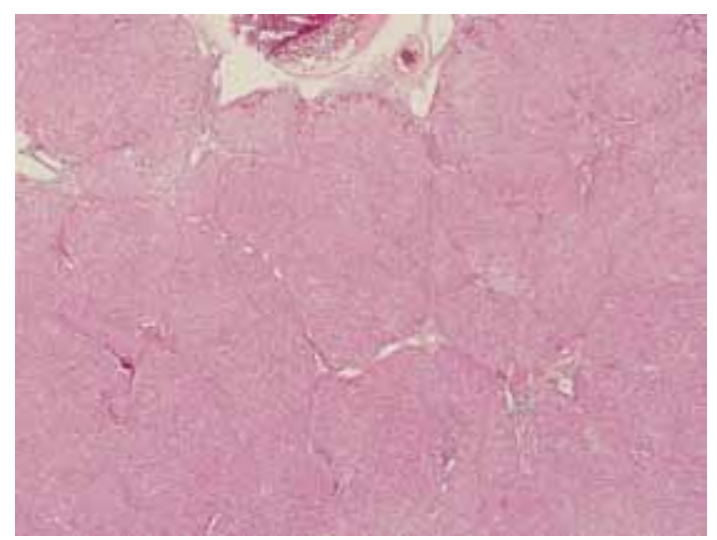

Figure 2. Group E (HE stained ×40)

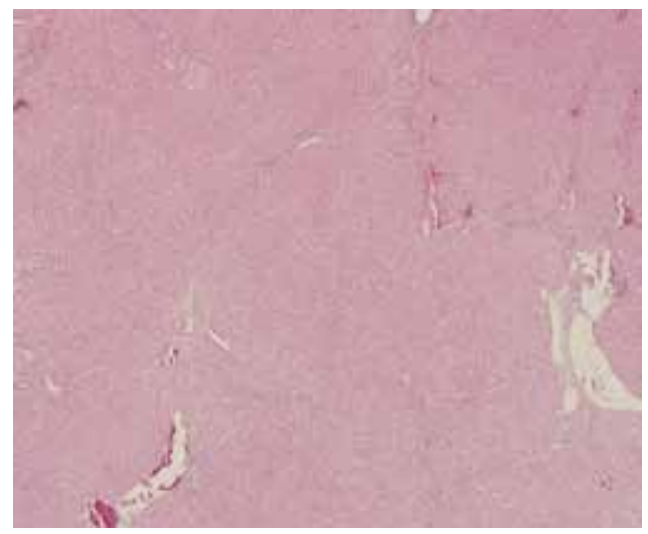

Figure 3. Group A (HE stained $\times 40)$

no significant $(P>0.05)$. Compared with group $\mathrm{E}$, IOD of group A and B was obviously lower $(P<0.05)$, however. Multiple comparisons between group $\mathrm{A}$ and $\mathrm{B}$, and the difference was considered to be no significant $(P>0.05)$, as given by Table 5 .

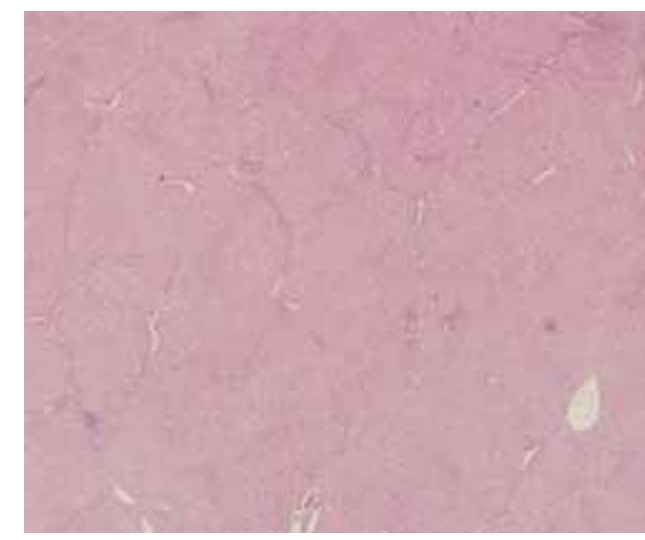

Figure 4. Group B (HE stained ×40)

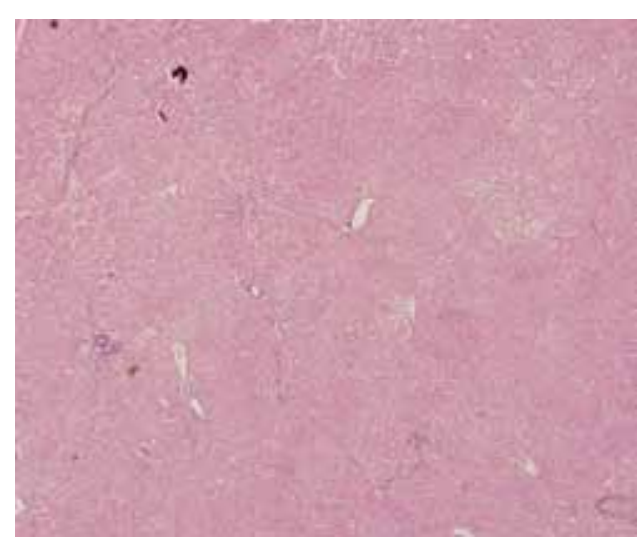

Figure 5. Group C (HE stained ×40)

Immumohistochemical Staining of CTGF: Yellowbrown granulated TIMP protein was observed in fibrous septum of hepatic portal area in rats at different groups. Compared group D and B with group E, the difference of IOD was considered to be no significant $(P>0.05)$. Compared with model control group, IOD of group A and $\mathrm{C}$ was obviously lower $(P<0.01)$. Multiple comparisons between group $\mathrm{A}$ and $\mathrm{C}$, the difference was considered to be no significant $(P>0.05)$, as given by Table 6 .

mRNA Expression of Hepatic CTGF, TIMP-1, procol- $\alpha 1$ and PC III at Different Groups was tested by Quantitative Real-Time PCR ( See Figure 7).

Result of Quantitative Test of Hepatic CTGF mRNA:

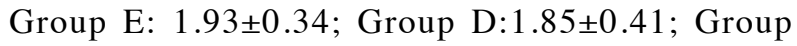
$\mathrm{C}: 1.97 \pm 0.76$; Compared group $\mathrm{C}$ and $\mathrm{D}$ with group $\mathrm{E}$, the difference was considered to be no significant. Group A: $1.13 \pm 0.34$; Group B: 1.21 \pm 0.37 ; Compared with group E, CTGF mRNA transcription of group A and B was 
obviously lower $(P<0.05)$.

Result of Quantitative Test of Hepatic TIMP-1 mRNA: Group E: $1.61 \pm 0.68$; Group B: $1.41 \pm 0.39$; Group D: $1.56 \pm 0.71$; Compared group $B$ and $D$ with group $E$, the difference was considered to be no significant. Group A: $0.95 \pm 0.11$; Group C: $0.84 \pm 0.12$; Compared with group E, Group A and B was obviously lower $(P<0.01)$.

Result of Quantitative Test of Hepatic procol- $\alpha 1$ mRNA at Different Groups: Group E: $2.17 \pm 0.45$; Group D: 2.15 \pm 0.46 ; Compared group $\mathrm{E}$ and $\mathrm{D}$, the difference was considered to be no significant. Group A: $1.05 \pm 0.25$; Group B: $1.53 \pm 0.77$; Group C: $1.57 \pm 0.25$; Compared with group E, Procol- $\alpha 1$ mRNA transcription of group A, B and $\mathrm{C}$ was obviously lower $(P<0.05)$. Multiple comparisons among group $\mathrm{A}, \mathrm{B}$ and $\mathrm{C}$, compared group $\mathrm{B}$ and $\mathrm{C}$, group A was obviously lower, the difference was considered to be significant $(P<0.05)$, and there was less difference between group $\mathrm{B}$ and $\mathrm{C}$.

Result of Quantitative Test of Hepatic PC III mRNA at Different Groups: Group E: 2.45 \pm 1.01 ; Group D: $2.47 \pm 0.52$; Compared group $\mathrm{E}$ with $\mathrm{D}$, the difference was considered to be no significant. Group A: $1.44 \pm 0.42$; Group B: $1.46 \pm 0.27$; Group C: $1.27 \pm 0.28$; Compared with group D and E, PC III mRNA transcription of group A, $\mathrm{B}$ and $\mathrm{C}$ was obviously lower $(P<0.05)$. The difference in multiple comparisons among group $\mathrm{A}, \mathrm{B}$ and $\mathrm{C}$ were considered to be no significant $(P>0.05)$.

\section{Discussion}

Successful model of hepatic fibrosis is a precondition to study therapy of hepatic fibrosis. DMN is chartered by hepatic genotoxicity, the hepatic model induced by DMN has advantages such as short modeling time, lower mortality, stable formation of hepatic fibrosis, less selfhealing trend without induction, and similar situation to earlier human hepatic fibrosis etc (Kuang et al., 2008). Its defect, however, DMN of high-dosage easily causes hepatonecrosis, and low-dosage is hard to formation of hepatic fibrosis. In this experiment, hepatic HE stained is done for dead rats, by microscope where we observe that formation of large pseudolobules, wide fibrous connective tissue septum, different degrees of hepatic degeneration and necrosis, which are similar to cirrhosis after necrosis. Typical hepatic fibrosis can be seen in the pathological sections of alive rats after interference, as well as pseudolobule in some sections.

HSC is the key cell during the formation of hepatic fibrosis, HSC is changed to be myofibroblast and a large number of ECM is synthesized by change of its phenotype and functions. However, TGF- $\beta 1$ is the best factor of promoting fiber formation for HSC (Yang et al., 2011; Xu et al., 2010).

Signal pathway of downstream TGF- $\beta /$ Sma is activated by TGF- $\beta$ and its sub-type, this effect is connected with hepatic fibrosis, cirrhosis and final formation of hepatocellular carcinoma. Thus, it is impossible to be the core target of drug therapies, signal pathways of TGF- $\beta 1$ stopped will be the great therapy for hepatic fibrosis (Giannelli et al., 2011). However, different target cells will be affected by TGF- $\beta 1$ and their biological effect is too complicated, the signal pathway of TGF- $\beta /$ Smad makes full use of physiological functions such as inhibition on inflammation, immunoregulation and promotion of cell growth etc, totally block of expression or inhibition on activity may cause unpredictable consequences (Yan et al., 2011). Therefore, it has great significance that more accurate and effective act on downstream pathway activated by TGF- $\beta 1$.

CTGF is a recently discovered important cytokine of promoting organ fibrosis. By induction of TGF- $\beta$, CTGF is produced by interstitial cells such as fibroblast etc, and in turn CTGF mediates the effect of TGF- $\beta$ on mesenchyme. As the cases that promotion of fibroblast proliferation, synthesis of collagen and extracellular matrix etc, this is the reason considering CTGF as biological functional medium of downstream of TGF- $\beta$. The study finds that abnormal high-expression of CTGF induced by TGF- $\beta$ always plays a key during hepatic fibrosis generation and development (Tache et al., 2011).

Matrix mealloproteiuases (MMP) plays crucial role during ECM degradation, and its activity is specially inhibited by TIMP where MMP activity is strongly inhibited by TIMP-1. TIMP-1 is induced and produced by TGF- $\beta$ and its signal pathways, and degradation of ECM by MMPs is stopped (Hemmann et al., 2011). Thus, TIMP-1 is considered as the downstream effect medium in biological function of TGF- $\beta 1$.

RNA interference is an excellent tool of silencing gene after transcription, which provides new way to effectively and specially regulate target cell's target gene expression down based on monitor on level of abnormal or exogenous genetic materials in organism and regulation on gene expression (Rettiq et al., 2012). This work focuses RNA dual interference on CTGF and TIMP-1, based on 2 ways including block of CTGF during formation of extracellular matrix (ECM) and removal of inhibition of TIMP-1 on EMC (to promote degradation), to keep away from the upstream of signal pathways of hepatic fibrosis and maintain their physiological functions. Meanwhile, it is able to block hepatic fibrosis signals of various upstream molecules due to CTGF and TIMP-1 are located at downstream intersection of more than one hepatic fibrosis signal pathways, which is a new idea of keeping hepatic fibrosis away.

In this study the results suggest that CTGF shRNA is of a factor obvious inhibition on mRNA expression of hepatic CTGF and procol- $\alpha 1$ in fibrotic rats , TIMP1 shRNA is of a factor obvious inhibition on mRNA expression of TIMP- 1 and procol- $\alpha 1$. Dual interference of psiRNA- GFP-Com of recombinant plasmid regulates hepatic expression of CTGF and TIMP-1 in rats down. Inhibition of psiRNA- GFP-Comon dual interference on procol- $\alpha 1 \mathrm{mRNA}$ expression is superior to that of single interference of psiRNA-GFP- CTGF and psiRNA-GFPTIMP-1, which is consistent with expression results of hepatic immumohistochemical staining of procol- $\alpha 1$ at different groups.

CTGF or TIMP-1 is taken as target gene and shRNA transcribed by plasmid is used, which inhibits the target gene expression, more important, inhibition on downstream ECM expression is achieved by inhibition on 
target gene, better inhibition will be observed if combined interference on CTGF or TMIPT-1 as target gene. This places a preparation to further find multi-link combined gene therapies for hepatic fibrosis.

\section{Acknowledgements}

Dr. Xin-En Huang is supported by Traditional Chinese Medicine Scientifi Research Project (LZ11091) and Jiangsu Province fourth stage "333 high- level Personnel Training Project” third levels of talent cultivating object.

\section{References}

Giannelli G, Mazzocca A, Fransvea E, et al (2011). Inhibiting TGF- $\beta 1$ signaling in hepatocelluar carcnoma. Biochim Biophys Acta, 1815, 214-23.

Hepatic fibrosis group of chinese society of hepatology (2002). consensus about evaluating hepatic fibrosis diagnosis and efficacy. Chinese J Hepatol, 10, 327-328.

Hemmann S, Graf J, Roderfeld M, et al (2007). Expression of MMPs and TIMPs in liver fibrosis-asystematic review with special emphasis on anti-fibrotic strategies. J Hepatol, 46, 955-75.

Jiang YF, Huang F, Zhang JJ, et al (2011). RNA interference targets on connective tissue growth factor and tissue inhibitor tissue inhibtor of metalloproteinase-1 in vitro. $J$ Clin Hepatol, 14, 246-8.

Jiang YF, Liu JQ, Zhang JJ, et al (2011). Construction of two different hepatic fibrosis-related cytokines shRNA recombinant plasmid. Chongqing Med, 40, 2341-2.

Jiang YF, Zhou DJ, Zhang JJ, et al (2012). Construction of dual RNA interference plasmid of hepatic fibrosis-related cytokines and study on transfection of hepatic stellate cells. J Clin Hepatol, 15, 516-9.

Kuang SL, Hu B (2008). Development of study on model of hepatic fibrosis in rats. Laboratory Animal Comparative Med, 28, 62-66.

Lee UE, Friedman SL (2011). Mechanisms of hepatic fibrogenisis. Best Pract Res Clin Gastroenterol, 25, 195-206.

Rettiq GR,Behlke MA (2012). Progress toward in vivo use of siRNA-II. Mol Ther, 20, 483-512.

Tache D,Boqdan F,Pisoschi C, et al (2011). Evidence for the involvement of TGF- $\beta 1$-CTGF axis in liver fibrogenisis secondary to hepatic viral infection. Rom J Morphol Embryol, 52, 409-12.

Xu LM (2010). Update on hepatic fibrosis research. J Clin Hepatol, 18, 563-565.

Yang J,Zheng J,Wu L, et al (2011). MDRG2 ameliorates hepatic fibrosis by inhibiting the TGF- $\beta 1 /$ Smad pathway and altering the MMP2/TIMP2 ratio in rats. PLoS One,6, 27710.

Yan X,Chen YG (2011). Smad7:not only a regulator,but also a cross-talk mediator of TGF- $\beta$ signaling. Biochem $J, \mathbf{4 3 4}$, $1-10$. 